\title{
Vinculação no Contexto Familiar: Relações entre Cônjuges, entre Pais e Filhos Adolescentes e entre Irmãos
}

\author{
Attachment in the Family Context: Relationships between Spouses, \\ between Parents and Adolescent Children and between Siblings
}

\author{
Raquel Geraldes*, Isabel Soares \& Carla Martins \\ Universidade do Minho, Braga, Distrito de Braga, Portugal
}

\begin{abstract}
Resumo
Tendo em vista a exploração das relações entre estilos de vinculação de membros de famílias e a sua associação com dimensões da vinculação na adolescência foram realizados dois estudos. O primeiro visou à validação psicométrica de uma sub-escala de avaliação da relação com o irmão, acrescentada ao instrumento Inventory of Parent and Peer Attachment (IPPA), evidenciando as qualidades psicométricas aceitáveis da mesma $(N=236)$. No segundo foram avaliados, através de três instrumentos de auto relato (incluindo a sub-escala validada), as relações e estilos de vinculação dos membros das famílias $(N=60)$. Conclusivamente, realça-se a concordância entre os estilos de vinculação, as correlações significativas entre dimensões de vinculação relativas a diferentes relações e a especificidade da relação entre irmãos neste contexto.

Palavras-chave: Vinculação, família, adolescentes, relações, pais-filhos
\end{abstract}

\begin{abstract}
In order to explore the relationships between attachment styles of family members and the association of those with dimensions of attachment in adolescence, two studies have been made. The first study aimed the psychometric validation of an evaluation sub-scale of siblings relationship added to the Inventory of Parent and Peer Attachment (IPPA) that demonstrated acceptable psychometric qualities $(N=236)$. The second study aimed, using three self-report instruments (with the validated sub-scale included), the evaluation of relationships and attachment styles of nuclear-family members $(N=60)$. In conclusion, it is highlighted the balance between family members' attachment styles, significant correlations between attachment dimensions that characterize different dyadic relationships and the specificity of the relationship among siblings in the family context.

Keywords: Attachment, family, adolescents, relationships, parents-children.
\end{abstract}

É no contexto familiar que se inscreve o desenvolvimento das relações de vinculação, a partir da matriz relacional em torno da prestação de cuidados. Se a díade criança-figura parental é o seu ponto de partida, desde os primeiros anos de vida, este processo desenvolvimental de natureza relacional amplia-se e estabelece-se com outros elementos no seio da família, tais como irmãos e avós, e, posteriormente, inclui outras relações emocionalmente significativas e duradouras que vão sendo construídas em outros contextos de vida (Day \& Padilla-Walker, 2009).

A investigação da vinculação no âmbito das relações entre irmãos direccionou-se, inicialmente, no sentido da possibilidade dos irmãos mais velhos poderem desempenhar, em situações de stress e/ou na ausência da figura materna, um papel de prestador de cuidados dos irmãos mais

*Endereço para correspondência: Escola de Psicologia, Universidade do Minho, Campus de Gualtar, Braga, Distrito de Braga, Portugal 4710-057. E-mail: raquelgeraldes@sapo.pt, isoares@psi.uminho.ptecmartins@ psi.uminho.pt novos (Ainsworth, 1989; Howe \& Rinaldi, 2004; Stewart, 1983). Estudos apontam para o facto dos irmãos mais velhos poderem funcionar, nomeadamente, como refúgio de segurança em momentos de ameaça/stress (Ainsworth, 1989; Stewart, 1983; Volling, 2001). No entanto, estes comportamentos entre irmãos parecem variar consoante a qualidade da relação que estabelecem com a figura materna: irmãos mais novos dirigem mais comportamentos de vinculação a irmãos mais velhos quando estes últimos são categorizados como seguros (Teti \& Ablard, 1989).

$\mathrm{O}$ facto de irmãos com idades aproximadas poderem funcionar mutuamente como companheiros de brincadeira, estabelecendo, ao longo do seu desenvolvimento, relações simétricas, cooperativas, recíprocas e confiantes, torna clara a possibilidade destas relações comportarem componentes de vinculação segura ou insegura, constituindo-se como vínculos afectivos (Ainsworth, 1989). Estas relações são, no entanto, influenciadas pelas relações de vinculação de cada um dos irmãos da díade (Teti \& Ablard, 1989). 
Buist, Dekovic, Meus e van Aken (2002) analisaram famílias integrando ambos os pais e, pelo menos, dois filhos entre os onze e os quinze anos. Com os adolescentes foi utilizado o Inventory of Parent and Peer Attachment (Armsden \& Greenberg, 1987), no qual foi introduzida uma folha de avaliação (com itens similares à folha de avaliação dos pais) referente à relação com o irmão, sendo os dados recolhidos em mais do que um momento. Os autores concluíram que ao longo da adolescência parece existir uma deterioração da qualidade das relações entre os adolescentes e os pais. Quanto às relações com os irmãos os padrões de desenvolvimento parecem ser mais complexos. O sexo do adolescente surge como uma variável que influencia a qualidade das relações no contexto da família nuclear: díades de irmãos do mesmo sexo surgem como mais próximas do que díades do sexo oposto, sendo que as díades do sexo feminino são aquelas que apresentam níveis mais altos de proximidade.

No contexto dos estudos relativos à concordância dos estilos de vinculação de irmãos criados na mesma família, pressupõe-se que: se os comportamentos parentais de prestação de cuidados são conduzidos pelas representações de vinculação estáveis dos pais, será de esperar que irmãos criados na mesma família experienciem comportamentos de prestação de cuidados semelhantes, moldando estas a qualidade das suas relações de vinculação com os pais (Kiang \& Furman, 2007; Samek \& Rueter, 2011). No seu Modelo de Concordância nas Representações de Vinculação dos Adolescentes aos Pais, Kiang e Furman (2007) propõem a existência de um ciclo intergeracional no qual as representações de vinculação dos pais afectam a qualidade da sua prestação de cuidados, a qual, por sua vez, afecta a relação de vinculação pais-filhos, e, desse modo, têm impacto nas representações mentais de vinculação que a criança constrói em relação a cada um dos pais. É esperado que estas representações sejam relativamente estáveis e que, durante a adolescência, progridam no sentido de uma representação generalizada da vinculação aos pais. Como este processo começa com a representação de vinculação dos próprios pais, que deverão ter um efeito similar nos diferentes filhos, este modelo preconiza a existência de uma concordância entre a representação de vinculação dos irmãos adolescentes aos pais.

Apesar de o modelo apresentado ter sido validado empiricamente, a investigação em torno da concordância das representações de vinculação entre irmãos não tem corroborado os resultados obtidos. No estudo conduzido com irmãos adolescentes para validação do modelo teórico apresentado, os autores não encontraram os níveis elevados de concordância que seriam de esperar de acordo com a proposta teórica, sendo que as variáveis 'sexo' e 'intervalo de idades entre os membros da díade', enquanto parasitas, não se mostraram determinantes dos resultados encontrados. Outras investigações apontam para níveis de concordância inferiores ao esperado. Numa meta-análise de dados provenientes de três grupos de investigação internacionais (van Ijzendoorn et al., 2000), foram analisados dados de 138 díades de irmãos entre os doze e os catorze meses. Os resultados sugeriram a existência de uma concordância significativa quando os dados eram aglutinados em apenas duas categorias de qualidade da vinculação (segura $v s$. insegura). A adopção da bidimensionalidade na avaliação da qualidade de vinculação dos irmãos foi também utilizada noutras investigações, conduzindo igualmente a níveis de concordância mais elevados do que aqueles que eram obtidos com a utilização das quatro categorias de qualidade de vinculação propostas por Ainsworth, Blehar, Waters e Wall (1978) e Main e Solomon (citado por Kiang \& Furman, 2007; O'Connor $\&$ Croft, 2001; Soares, Martins, \& Tereno, 2007). No entanto, grande parte dos estudos levados a cabo aponta para a existência de uma concordância modesta a moderada nas representações de vinculação de irmãos criados na mesma família (Kiang \& Furman, 2007; O'Connor \& Croft, 2001). Estes resultados encontram-se muito aquém do que seria teoricamente esperado, nomeadamente se tivermos em conta o modelo teórico proposto por Kiang e Furman (2007).

Este corpus de resultados proporcionou a emergência de uma nova linha de investigação dedicada à exploração de possíveis factores que possam explicar a não-concordância entre os estilos de vinculação de irmãos criados na mesma família. As condicionantes ambientais não-partilhadas pelos irmãos, surgiram então, como possibilidades teoricamente viáveis (Kiang \& Furman, 2007; O'Connor \& Croft, 2001; O'Connor, Croft, \& Steele, 2000). No âmbito destes "nonshared environmental factors" (O'Connor et al., 2000) foram indicadas a possibilidade de variação das práticas parentais consoante as experiências dos pais com o primeiro filho ou como resultado do nascimento de um segundo filho, a possibilidade de a interaç̧ão entre as representações mentais de vinculação dos pais com as características da criança produzir padrões únicos de vinculação nos filhos, e a possibilidade de existência de uma representação parental única a cada filho que determine as práticas em direcção ao mesmo (Kiang \& Furman, 2007; van Ijzendoorn et al., 2000). O temperamento, as percepções maternas da similaridade entre os filhos e as experiências da mãe com cada filho, são também indicados como possíveis condicionantes destes índices de concordância (MacCarthey \& Diggins citado por van Ijzendoorn et al., 2000). Numa outra perspectiva, considerando a possibilidade de os pais se comportarem de forma similar com ambos os filhos, estes últimos podem valorizar diferentemente as suas relações com a mãe e com o pai, o que, com a colisão das duas representações de vinculação numa única generalizada, poderá contribuir para não-concordância entre os estilos de vinculação dos filhos adolescentes do mesmo casal (Kiang \& Furman, 2007).

Concluindo, a concordância entre os estilos de vinculação dos irmãos surge como uma temática muito complexa e que não poderá ser melhor explorada sem um olhar 
Geraldes, R., Soares, I. \& Martins, C. (2013). Vinculação no Contexto Familiar: Relações entre Cônjuges, entre Pais e Filhos Adolescentes e entre Irmãos.

atento a todas as variáveis que nela possam exercer alguma influência (Fortuna, Roisman, Haydon, Groh, \& Holland, 2011). Assim, sendo os estilos de vinculação dos irmãos alicerçados no contexto das relações familiares deverão estas ser alvo de uma análise atenta.

Marvin e Stewart introduziram, em 1990, o conceito de modelo interno dinâmico partilhado, iniciando as propostas de alargamento do conceito de vinculação ao contexto familiar, ou seja, uma representação mental das relações partilhada por todos os membros da família, enquanto parte integrante de um todo. Já Bretherton, em 1985, havia considerado os "scripts" (que designam quem faz o quê, quando, como e porquê no contexto familiar) como um dos aspectos dos Modelos Internos Dinâmicos preconizados por Bowlby (1969/1982).

Nesta linha, Byng-Hall (1995) propôs o conceito de "family script", referindo-se este às expectativas partilhadas que a família tem acerca de como os papéis familiares deverão ser desempenhados em vários contextos, incluindo o de procura e prestação de cuidados. Segundo o autor, esta estrutura mental partilhada permite "a mutually held working model of how all the various attachments operate" (Byng-Hall \& Stevenson-Hinde, 1991, p. 201). Em 1995, o mesmo autor sugere ainda o conceito de "secure family base", afirmando que esta característica familiar "provides a reliable network of care that gives every member, of whatever age, a sufficient sense of security to explore and develop" (Byng-Hall, 1995, p. 19).

As várias relações diádicas constituintes desta rede familiar irão influenciar-se mutuamente (Stevenson-Hinde, 1990), com a finalidade de criar um ambiente seguro e confiável para os seus membros (Waters \& Cummings, 2000). Sumariamente, o conjunto de todas as relações diádicas familiares, em interacção umas com as outras, proporcionará um conhecimento mútuo dos papéis desempenhados por cada membro no contexto de todas essas relações, o que contribui para que o sistema, enquanto um todo, providencie (ou não) uma base segura comum a todos os seus elementos.

Para Ainsworth et al. (1978), a partilha de experiências ao longo do tempo, tal como acontece na família, promove semelhanças no modo como se percepcionam as situações e no sistema de valores, influenciando o entendimento mútuo, muitas vezes alheio à comunicação explícita.

Estas sugestões são coerentes com a de Bowlby de que

.. . human beings of all ages are happiest and able to deploy their talents to their best advantage when they are confident that, standing behind them, there are one or more trusted . . . can be considered as providing his companion with a secure base from which to operate. (Bowlby, 1969/1982, p. 103)

Apesar da questão da vinculação na família ter já sido discutida e equacionada por inúmeros autores, a investigação acerca desta temática permanece ainda muito escassa (Riggs \& Riggs, 2011), não sendo conhecidos quaisquer estudos em Portugal neste âmbito.
No que à concordância entre os estilos de vinculação dos membros do casal heterossexual diz respeito, a evidência empírica internacional sugere que mulheres (in) seguras tendam a relacionar-se intimamente com homens (in)seguros (Batgos \& Leadbeater, 1994; Mehta, Cowan, \& Cowan, 2009; van Ijzendoorn, 1992; van Ijzendoorn \& Bakermans-Kranenburg, 1996). Numa meta-análise de cinco estudos efectuados a este respeito (van Ijzendoorn \& Bakermans-Kranenburg, 1996), a correspondência entre o estilo de vinculação de marido e mulher surgiu como significativa.

Quanto à possível concordância entre os estilos de vinculação dos pais e dos filhos, Van Ijzendoorn, em 1992 , analisou quatro estudos e concluiu que existiam fortes concordâncias entre a representação mental de vinculação dos pais e a sua relação de vinculação com os filhos, o que vem confirmar a hipótese da transmissão intergeracional da vinculação. Apesar de os filhos tenderem a manter relações de vinculação concordantes com ambos os pais (Sullivan et al., 2006), há dados que apontam para o facto de o sexo dos primeiros poder influenciar a qualidade da vinculação aos pais (van Ijzendoorn et al., 2000). No estudo de Sullivan et al. (2006), o sexo dos filhos surgiu como significativamente associado à qualidade da vinculação aos pais: encontrou-se concordância na qualidade de vinculação das raparigas a ambos os pais mas não dos rapazes, encontrando-se estes mais seguros aos pais do que as primeiras. Outros estudos já haviam apontado neste sentido, indicando que as relações pais-filhos são únicas, quando comparadas com as relações pais-filhas, mães-filhos ou mães-filhas (Sullivan et al., 2006). No entanto, o facto de outras investigações apontarem para que "as relações de vinculação entre filhos e pais parecem ser de alguma forma congruentes" (Sullivan et al., 2006, p. 380) vem reforçar a necessidade de se conduzirem mais estudos que possam esclarecer o modo como as relações de vinculação se organizam no contexto da família.

Nesse sentido, o presente estudo visa examinar as relações entre os estilos de vinculação dos membros de uma mesma família constituída por: mãe, pai e dois filhos adolescentes. Para a realização deste estudo foi levada a cabo uma investigação prévia tendo em vista a validação da sub-escala do Inventory of Parent and Peer Attachment (IPPA) relativa à avaliação das relações entre irmãos.

\section{Estudo I - Validação Psicométrica da Sub-Escala do IPPA Relativa aos Irmãos}

O Inventory of Parent and Peer Attachment (IPPA) inclui, originalmente, as escalas de avaliação dos comportamentos, cognições e estados emocionais que envolvem a confiança, a compreensão e respeito mútuo, a acessibilidade e responsividade das figuras (mãe, pai e melhor amigo), estados emocionais como sejam a raiva, a irritação e o ressentimento dirigido a essas figuras e, ainda, a desvinculação ou o isolamento da relação (Neves, Soares, 
\& Silva, 1999). Este instrumento tem sido amplamente utilizado no panorama nacional e internacional da investigação debruçada sobre a vinculação na adolescência, o que tem evidenciado a sua pertinência e utilidade na busca de um melhor entendimento do fenómeno e confirmação empírica da teoria da vinculação (Cunha, 2003).

A lacuna teórico-empírica ao nível das relações entre irmãos, abordadas à luz da Teoria de Vinculação, justifica e suporta a possibilidade de se incluir, no instrumento, uma escala de avaliação das relações estabelecidas no contexto da fratria. Com esta possibilidade, o IPPA torna-se um instrumento de avaliação da vinculação na adolescência que integra grande parte das relações significativas mantidas nesta fase desenvolvimental, permitindo, por si só, o acesso a uma compreensão quase holística das relações de vinculação do adolescente.

A versão inicial do IPPA (Inventory of Parent and Peer Attachment, então designado como IAA - Inventory of Adolescent Attachment) foi apresentada por Greenberg em 1983 e era constituída por duas escalas que avaliavam a qualidade das relações afectivas do adolescente com pais e amigos (Greenberg, Siegel, \& Leitch, 1983). Já em 1987, Armsden e Greenberg apresentaram uma versão revista do IPPA, sendo este instrumento revisto constituído por 3 escalas (com 25 itens cada), nas quais os adolescentes discriminavam, através de uma escala tipo Likert (1-Nunca; 5-Sempre), a frequência com que experienciavam determinados sentimentos em relação à mãe, ao pai e ao melhor amigo. As três escalas revelaram, no estudo referido, um coeficiente de consistência interna superior a 0,80 . Em Portugal, o primeiro estudo de adaptação foi efectuado por Geada (1990) e, posteriormente em outros estudos (Cunha, 2003; Neves et al., 1999).

Buist et al. (2002) foram pioneiros na inclusão de uma escala de avaliação da relação com o irmão no IPPA, com uma amostra de adolescentes com idades compreendidas entre os 11 e os 15 anos. Os autores revelaram a existência de uma correspondência entre os factores extraídos desta nova escala (comunicação, confiança e alienação) e os já existentes nas escalas relativas a mãe e pai, não discriminando, no entanto, as análises efectuadas com respeito à utilização deste instrumento na avaliação da qualidade da relação entre irmãos.

Em Portugal, não foram encontrados estudos que avaliem a relação entre irmãos na adolescência, no quadro de referência da teoria de vinculação. Esta lacuna realça a pertinência do presente estudo psicométrico como contributo metodológico para esta área, o que permitirá subsequentemente a testagem de novas hipóteses de investigação na área da vinculação na família nuclear e na adolescência.

\section{Método}

\section{Participantes}

Participaram, neste estudo, 236 adolescentes com idades compreendidas entre os 13 e os 18 anos $(M=15,25$; $D P=1,80), 52,5 \%$ dos quais do sexo feminino $(n=124)$.
Os irmãos dos adolescentes tinham idades compreendidas entre os 1 e os 26 anos $(M=15,31 ; D P=4,52)$, sendo $56,36 \%$ do sexo feminino $(n=133)$.

\section{Instrumento}

No presente estudo, foi utilizada a versão portuguesa do IPPA (Inventário sobre Vinculação na Adolescência; Neves et al., 1999), com três escalas (de 25 itens cada) referentes às figuras de pai, mãe e melhor amigo, avaliadas numa escala de tipo Likert de cinco pontos (1- Nunca, 5-Sempre). A este instrumento original foi acrescentada uma nova folha de avaliação, similar às referentes ao pai e mãe, mas que solicita ao sujeito a avaliação da sua relação com o irmão, tal como efectuado no estudo de Buist et al. (2002).

\section{Procedimentos}

A recolha de dados foi efectuada pelo método de amostragem de conveniência, em duas escolas públicas (uma do ensino básico e outra do ensino secundário), pertencentes ao distrito do Porto, em contexto de sala de aula. Após a autorização dos concelhos executivos das duas escolas, foram contactados alguns directores de turma com o intuito de explicar os objectivos e procedimentos do estudo. Foram agendadas as visitas para recolha de dados, sendo, nestes casos, explicado directamente aos alunos o objectivo do estudo e a relevância da sua participação. Foi solicitado o consentimento informado dos progenitores para a participação, no estudo, dos adolescentes menores. Em algumas turmas, na impossibilidade de a recolha ser efectuada directamente pela equipa de investigação, foram dadas instruções ao professor, sendo ele mesmo a efectuar a recolha.

\section{Resultados Psicométricos: Sensibilidade, Validade e Fidelidade}

Com o objectivo de analisar as qualidades psicométricas da escala de avaliação da relação entre irmãos do IPPA foram realizadas análises relativas à validade e fidelidade dos resultados obtidos junto da amostra já caracterizada. Na execução das análises foi utilizado o Statistical Package for Social Sciences (IBM SPSS - versão 19.0).

Ao nível da validade do instrumento, foi efectuada uma Análise de Componentes Principais, seguida de rotação Varimax, através da qual foram extraídos dois factores explicativos de $52,5 \%$ da variância total. O factor 1 explica $44,6 \%$ da variância global e o factor 2 explica $7,9 \%$ da mesma (cf. Tabela 1).

$\mathrm{O}$ factor 1 inclui os itens $1,2,4,5,6,7,12,13,15$, $16,20,21,22,24$ e 25 , os quais avaliam comportamentos, cognições e estados emocionais indicadores de confiança, comunicação, respeito mútuo, cumplicidade, disponibilidade e responsividade, recebendo, por isso, a designação de "Proximidade".

No factor 2 encontram-se incluídos os itens 3, 8, 9, 10, $11,14,17,18$ e 23 , que avaliam comportamentos, cognições e estados emocionais indicadores de distanciamento 
Geraldes, R., Soares, I. \& Martins, C. (2013). Vinculação no Contexto Familiar: Relações entre Cônjuges, entre Pais e Filhos Adolescentes e entre Irmãos.

Tabela 1

Loadings dos Itens nos Dois Factores, Comunalidades e Percentagem da Variância Explicada por Cada Factor

\begin{tabular}{|c|c|c|c|}
\hline Itens & $\mathrm{F} 1$ & $\mathrm{~F} 2$ & Comunalidades \\
\hline 1 - O meu irmão respeita os meus sentimentos & $\mathbf{0 , 7 3}$ & $-0,26$ & 0,60 \\
\hline 2 - Acho que o meu irmão é um bom irmão & $\mathbf{0 , 6 0}$ & 0,35 & 0,48 \\
\hline 3 - Gostava de ter um irmão diferente do que tenho & $-0,39$ & 0,63 & 0,55 \\
\hline 4- O meu irmão aceita-me tal como eu sou & 0,64 & $-0,30$ & 0,49 \\
\hline $\begin{array}{l}5 \text { - Gosto sempre de saber a opinião do meu irmão sobre as coisas } \\
\text { que são importantes para mim }\end{array}$ & $\mathbf{0 , 8 0}$ & 0,13 & 0,66 \\
\hline 6 - Acho inútil dar a conhecer os meus sentimentos ao meu irmão & $-0,54$ & 0,39 & 0,45 \\
\hline $\begin{array}{l}7 \text { - Mesmo quando estou fora de mim sou capaz de escutar } \\
\text { o que o meu irmão tem a dizer }\end{array}$ & 0,75 & $-0,24$ & 0,61 \\
\hline 8 - Tenho vergonha ou acho patetice falar dos meus problemas ao meu irmão & $-0,40$ & $\mathbf{0 , 5 1}$ & 0,42 \\
\hline 9 - O meu irmão espera demasiado de mim & 0,24 & 0,39 & 0,21 \\
\hline 10 - Perco a cabeça facilmente com o meu irmão & $-0,40$ & 0,64 & 0,55 \\
\hline 11 - Fico fora de mim mais frequentemente do que o meu irmão pensa & $-0,30$ & 0,61 & 0,46 \\
\hline $\begin{array}{l}12 \text { - Quando conversamos sobre qualquer assunto o meu irmão tem } \\
\text { em devida conta as minhas opiniões }\end{array}$ & $\mathbf{0 , 5 8}$ & $-0,05$ & 0,34 \\
\hline 13 - O meu irmão confia na minha capacidade de ajuizar as coisas & $\mathbf{0 , 7 0}$ & $-0,18$ & 0,53 \\
\hline 14- Não aborreço o meu irmão com os meus problemas, pois ele já tem os dele & $-0,11$ & $\mathbf{0 , 4 1}$ & 0,18 \\
\hline 15 - O meu irmão ajuda-me a compreender-me melhor a mim própria & $\mathbf{0 , 8 2}$ & $-0,24$ & 0,71 \\
\hline 16 - Costumo falar ao meu irmão dos meus problemas ou complicações & 0,76 & $-0,14$ & 0,59 \\
\hline 17 - O meu irmão irrita-me & $-0,44$ & 0,67 & 0,64 \\
\hline 18 - O meu irmão não me dá muita atenção & 0,04 & $\mathbf{0 , 6 0}$ & 0,36 \\
\hline 19 - O meu irmão ajuda-me a falar das minhas próprias dificuldades & $\mathbf{0 , 8 2}$ & $-0,11$ & 0,68 \\
\hline 20 - O meu irmão compreende-me & $\mathbf{0 , 8 4}$ & $-0,24$ & 0,75 \\
\hline 21 - Quando me irrito com qualquer coisa o meu irmão procura ser compreensivo & $\mathbf{0 , 8 0}$ & $-0,17$ & 0,68 \\
\hline 22 - Confio no meu irmão & $\mathbf{0 , 7 3}$ & $-0,32$ & 0,63 \\
\hline 23 - O meu irmão não compreende o que é a minha vida & $-0,15$ & $\mathbf{0 , 5 3}$ & 0,30 \\
\hline $\begin{array}{l}24 \text { - Posso contar com o meu irmão quando preciso de me ver livre } \\
\text { de algum peso que tenho dentro de mim }\end{array}$ & $\mathbf{0 , 8 1}$ & 0,13 & 0,68 \\
\hline $\begin{array}{l}25 \text { - Quando o meu irmão percebe que tenho alguma coisa que me aborrece } \\
\text { pergunta-me sempre o que tenho }\end{array}$ & $\mathbf{0 , 7 3}$ & $-0,16$ & 0,56 \\
\hline Variância Explicada & $44,6 \%$ & $7,9 \%$ & \\
\hline Alpha de Cronbach & 0,87 & 0,76 & \\
\hline
\end{tabular}

emocional, irritabilidade, isolamento e desvinculação, sendo-lhe, por este motivo, atribuída a designação de "Distanciamento"

Através da análise do coeficiente de Spearman foi possível concluir a existência de uma correlação negativa significativa entre os dois factores, $r_{s p}=-0,59, p<0,001$. Os coeficientes de alpha de Cronbach para ambos os factores, evidenciaram bons níveis de consistência interna: elevado no factor $1(\boldsymbol{\alpha}=0,87)$ e aceitável no factor $2(\boldsymbol{\alpha}$ $=0,76$ ). 


\section{Efeito das Idades dos Irmãos nos Resultados \\ Psicométricos Obtidos}

Dada a diversidade de idades dos irmãos foram efectuadas análises no sentido de aceder ao efeito das mesmas na estrutura factorial da sub-escala. Considerando apenas os adolescentes com irmãos com idades compreendidas entre os 13 e 18 anos, a amostra geral foi dividida em dois grupos: (a) irmãos mais velhos com irmãos mais novos entre os 13 e os 18 anos $(n=80)$; e (b) irmãos mais novos com irmãos mais velhos entre os 13 e os 18 anos $(n=85)$.

Os resultados revelaram a existência de uma correspondência entre as análises efectuadas nas duas sub-amostras. Em ambas, recorrendo à Análise de Componentes Principais com rotação Varimax, foram extraídos dois factores. Foi utilizado o mesmo procedimento analítico da amostra original. Este resultado confirma e reifica as qualidades psicométricas do instrumento independentemente da posição na fratria do irmão avaliado (i.e., mais novo ou mais velho).

\section{Estudo II - Relações de Vinculação no Contexto Familiar}

\section{Método}

\section{Participantes}

Foram avaliadas 60 famílias (constituídas por pai, mãe e dois filhos, entre os 13 e os 18 anos). Quanto ao sexo dos filhos, $61,67 \%$ das famílias continham dois irmãos de sexos opostos $(n=37), 23,3 \%$ eram constituídas por duas adolescentes do sexo feminino $(n=14)$ e em $15 \%$ dos casos, os irmãos eram do sexo masculino $(n=9)$. Os irmãos mais novos tinham em média 14,57 anos $(D P=$ $1,16)$ e os mais velhos $17,08(D P=1,14)$. As mães tinham em média 43,62 anos $(D P=3,90)$ e os pais 45,95 anos $(D P=4,07)$. Dos 60 pais participantes $21,7 \%$ concluíram o $1^{\circ}$ ciclo $(n=13), 30 \%$ finalizaram o $2^{\circ}$ ciclo $(n=18)$, $18,3 \%$ terminaram o $3^{\circ}$ ciclo $(n=11), 11,7 \%$ frequentaram o Ensino Secundário $(n=7)$ e 18,3\% apresentavam formação superior ( $n=11)$. Quanto à figura materna, $8,3 \%$ das mães participantes concluíram o $1^{\circ}$ ciclo $(n=5), 25 \%$ terminaram o $2^{\circ}$ ciclo $(n=15), 28,3 \%$ finalizaram o $3^{\circ}$ ciclo $(n=17), 23,3 \%$ findaram o Ensino Secundário $(n=14) \mathrm{e}$ $15 \%$ apresentavam formação superior $(n=9)$.

\section{Instrumentos}

Foram utilizados no estudo um questionário sociodemográfico para caracterização da amostra, a Escala de Vinculação para Adultos (EVA - versão de auto-avaliação; Canavarro, 1997), o Inventário sobre Vinculação para a Infância e Adolescência (IVIA - versão de auto-avaliação; Carvalho, Soares, \& Baptista, 2013) e o Inventário sobre Vinculação na Adolescência (IPPA; Neves et al., 1999).

Avaliação da Vinculação nos Adultos. A Escala de Vinculação para Adultos (EVA; Canavarro, 1997; Collins \& Read, 1990) foi utilizada com o intuito de avaliar as percepções de estilos de vinculação do pai e da mãe de cada família. Esta escala foi construída por Collins e Read (Adult Attachment Scale-AAS - R; 1990) e adaptada para a população portuguesa por Canavarro (1997), permitindo avaliar a qualidade de vinculação segundo as categorias: segura, preocupada, desligada e amedrontada. Constituída por 18 itens, respondidos através de uma escala tipo Likert (1- Nada característico em mim; 5- Muito característico em mim), a escala revelou boas capacidades psicométricas na sua aplicação à população portuguesa (Canavarro, Dias, \& Lima, 2006), sendo o alpha de Cronbach total da escala de 0,81 . A cotação do instrumento foi efectuada através do cálculo das pontuações médias obtidas nos itens de cada categoria.

Avaliação da Vinculação em Adolescentes. O Inventário sobre Vinculação para a Infância e Adolescência (IVIA; Carvalho et al., 2013), constituído por 37 itens, foi utilizado para categorização dos irmãos adolescentes quanto ao seu estilo de vinculação: segura, ansiosa ou evitante. No instrumento é pedido aos adolescentes para avaliarem a frequência com que experienciam cada pensamento ou comportamento descrito, de acordo com uma escala tipo Likert (1-Nunca; 5-Sempre). Os coeficientes de consistência interna encontrados pelos autores originais do instrumento foram superiores a 0,70 (Carvalho et al., 2013). A cotação do instrumento foi efectuada, tal como no estudo de validação, através do cálculo do somatório das pontuações obtidas nos itens de cada escala.

Foi ainda utilizada a versão portuguesa do Inventário sobre Vinculação na Adolescência (IPPA; Neves et al., 1999), cujas características já foram descritas na fundamentação e à qual foi acrescentada uma nova folha de avaliação, similar às referentes ao pai e mãe, mas que solicita aos adolescentes que avaliem a sua relação com o irmão. Os resultados do estudo psicométrico foram apresentados no Estudo I.

\section{Procedimentos}

A recolha de dados foi efectuada, através do método de amostragem de conveniência, em duas escolas públicas (uma do ensino básico e outra do ensino secundário), pertencentes ao distrito do Porto. Foi solicitado previamente o consentimento informado dos progenitores para a participação, no estudo, dos irmãos menores. O Comité de Ética do Centro de Investigação em Psicologia da Escola de Psicologia da Universidade do Minho conferiu parecer positivo à realização do estudo.

Os instrumentos (agrupados por famílias) foram entregues a um dos irmãos no contexto escolar, sendo recolhidos quinze dias depois. A confidencialidade e anonimato dos dados foram garantidos de duas formas: os instrumentos de cada membro da família foram entregues em envelopes, sendo dadas indicações para que estes fossem selados no final do preenchimento; a cada família foi atribuído um número, garantindo a correspondência entre os instrumentos de todos os membros. Estas instruções, claramente expressas no cabeçalho dos instrumentos, tinham como objectivo prevenir a contaminação dos dados no seio das 
Geraldes, R., Soares, I. \& Martins, C. (2013). Vinculação no Contexto Familiar: Relações entre Cônjuges, entre Pais e Filhos Adolescentes e entre Irmãos.

famílias, no entanto, a garantia da não-contaminação não pôde ser assegurada. As análises de dados foram efectuadas com recurso ao Statistical Package for the Social Sciences - IBM SPSS 19.0.

\section{Resultados}

\section{Estilos de Vinculação dos Membros da Família}

Os dados relativos aos adolescentes revelaram algumas discrepâncias na distribuição pelos estilos de vinculação dos irmãos: $71,7 \%$ dos irmãos mais velhos e $88,3 \%$ dos irmãos mais novos são seguros; $15 \%$ e $5 \%$ dos irmãos mais velhos e mais novos, respectivamente, são ansiosos/ ambivalentes; e, $13,3 \%$ dos irmãos mais velhos e $6,7 \%$ dos irmãos mais novos são evitantes (cf. Figura 1).

A análise das frequências ao nível dos membros dos casais revelou uma distribuição similar em ambos os membros: $60 \%$ dos pais e mães são seguros; $26,7 \%$ são evitantes; $8,3 \%$ das mães e $10 \%$ dos pais são preocupados; e, $5 \%$ das mães e $3,3 \%$ dos pais são amedrontados (cf. Figura 1- Descrição dos Estilos de Vinculação dos Membros da Família).

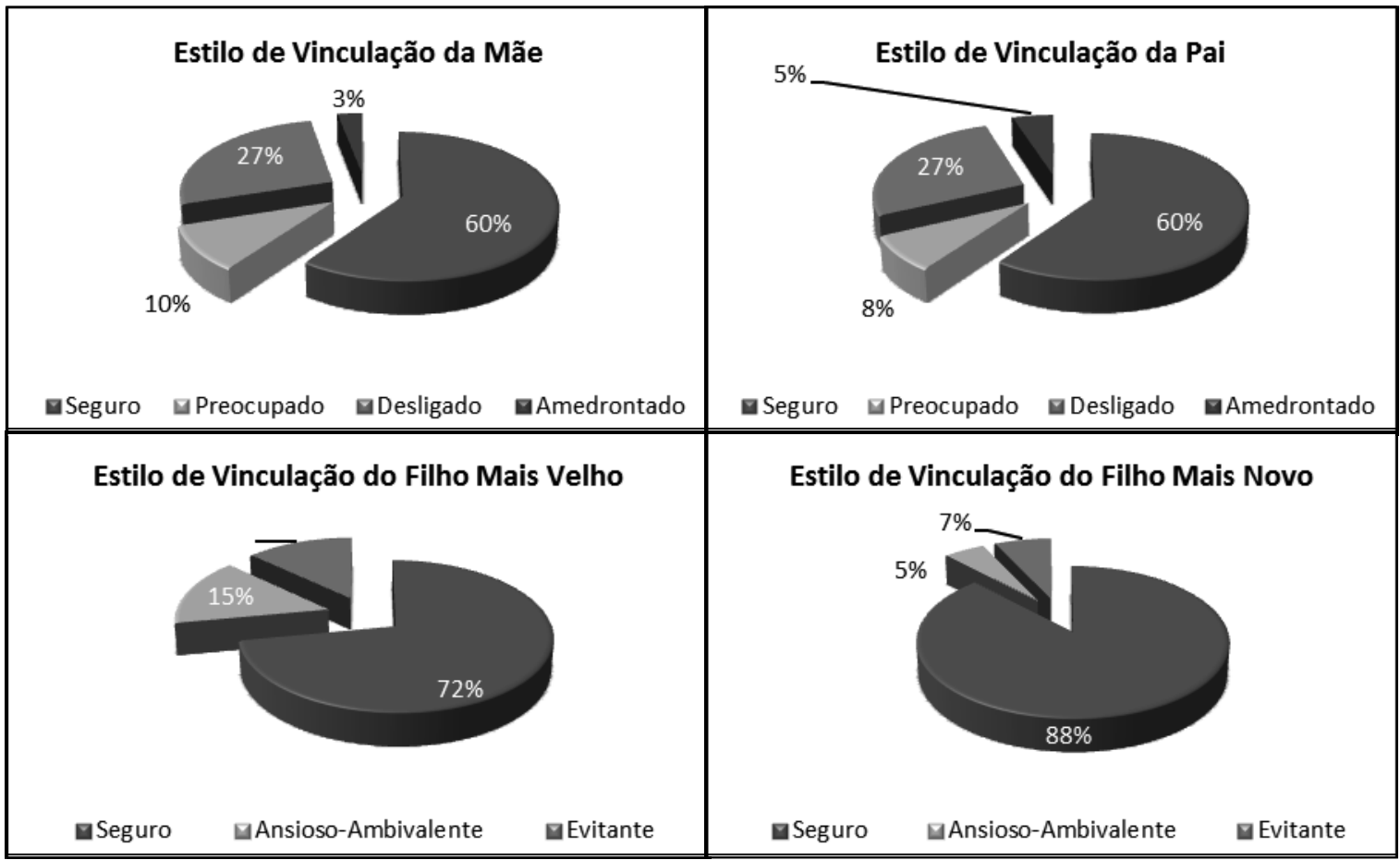

Figura 1. Descrição dos estilos de vinculação dos membros da família.

\section{Concordância entre os Estilos de Vinculação dos Membros da Família}

Com o objectivo de verificar a existência de uma associação entre os estilos de vinculação dos irmãos, entre os estilos de vinculação dos pais e dos irmãos e, por fim, entre os estilos de vinculação dos pais, foram utilizadas as classificações dicotómicas dos estilos de vinculação (seguro/inseguro) de forma a tornar os resultados mais consistentes.

Quanto aos estilos de vinculação dos irmãos, quando analisados os dados relativos à categorização segundo as principais dimensões da vinculação (segurança/insegurança), encontrou-se uma associação estatisticamente significativa, $\chi^{2}(2)=7,25, p=0,007$.

No caso das díades de irmãos, a concordância na segurança do estilo de vinculação mostrou-se claramente maioritária, quando comparada com as outras combinações possíveis. Em $68,3 \%(\mathrm{n}=41)$ das díades ambos os membros eram seguros enquanto apenas $8,3 \%(n=5)$ das díades integravam dois adolescentes inseguros. Em 20\% $(\mathrm{n}=12)$ das duplas de irmãos o mais novo era seguro e o mais velho inseguro e nos restantes $3,3 \%(n=2)$ acontecia o inverso.

Quanto à análise dos estilos de vinculação de pais (mãe e pai independentemente) e filhos (irmão mais velho e irmão mais novo independentemente), existe uma associação marginalmente significativa entre o estilo de vinculação da figura materna e o do irmão mais velho, $\chi 2(1)=3,50, \mathrm{p}$ $=0,061$, entre o estilo de vinculação da mãe e o do irmão mais novo, $\chi^{2}(1)=3,26, p=0,071$, e entre o estilo de vinculação do pai e o do irmão mais velho, $\chi^{2}(1)=3,50$, $\mathrm{p}=0,061$. Não existe uma associação estaticamente significativa entre o estilo de vinculação da figura paterna e o do irmão mais novo, $\chi 2(1)=0,03, p=0,87$ (cf. Tabela 2).

$\mathrm{Na}$ Tabela 2 encontram-se as percentagens relativas a cada combinação segurança/insegurança das díades mãe-irmão mais velho, mãe-irmão mais novo, pai-irmão mais velho e pai-irmão mais novo. 
Psicologia: Reflexão e Crítica, 26(4), 799-808.

Tabela 2

Frequência e Percentagens das Associações entre Segurança/Insegurança de Pais e Irmãos

\begin{tabular}{cccccccccc}
\hline & & \multicolumn{3}{c}{ Irmão Mais Velho } & \multicolumn{3}{c}{ Irmão Mais Novo } \\
\cline { 3 - 10 } & & \multicolumn{2}{c}{ Seguro } & \multicolumn{2}{c}{ Inseguro } & \multicolumn{2}{c}{ Seguro } & \multicolumn{2}{c}{ Inseguro } \\
\hline \multirow{3}{*}{ Mãe } & $\mathrm{n}$ & $\%$ & $\mathrm{n}$ & $\%$ & $\mathrm{n}$ & $\%$ & $\mathrm{n}$ & $\%$ \\
& Seguro & 29 & 48,3 & 7 & 11,7 & 34 & 56,7 & 2 & 3,3 \\
& Inseguro & 14 & 23,3 & 10 & 16,7 & 19 & 31,7 & 5 & 8,3 \\
\multirow{2}{*}{ Pai } & Seguro & 29 & 48,3 & 7 & 11,7 & 32 & 53,3 & 4 & 6,7 \\
& Inseguro & 14 & 23,3 & 10 & 16,7 & 21 & 35 & 3 & 5 \\
\hline
\end{tabular}

Verifica-se que as díades mãe/pai-irmão mais velho/ novo são principalmente constituídas por membros seguros, sendo esta a combinação mais frequente e à qual se segue a combinação mãe/pai inseguro e irmãos mais velho/ novo seguro. A combinação mãe/pai seguro e irmão mais velho/novo inseguro é aquela menos frequente excepcionando no caso das díades pai-irmão mais novo, sendo estas aquelas que não demonstraram uma associação estatisticamente significativa entre si (Tabela 1).

$\mathrm{Na}$ análise dos estilos de vinculação de mãe e pai foi encontrada uma associação significativa entre a percepção dos estilos de vinculação dos pais e das mães, $\chi^{2}(1)=$ $8,44, \mathrm{p}=0,004$. Em 45\% ( $\mathrm{n}=27)$ dos casais ambos os membros eram seguros e $25 \%(\mathrm{n}=15)$ das díades eram constituídas por pai e mãe inseguros. Em 15\% $(n=9)$ das famílias a mãe percepcionava o seu estilo de vinculação como seguro e o pai como inseguro e nos restantes $15 \%$ $(\mathrm{n}=9)$ acontecia o inverso.

\section{Discussão}

O primeiro objectivo do estudo incidiu na validação psicométrica da sub-escala do IPPA relativa aos irmãos. Os resultados deste primeiro estudo revelam qualidades psicométricas aceitáveis referentes à escala do IPPA que avalia as dimensões cognitivas, afectivas e comportamentais da relação entre irmãos sob o ponto de vista da teoria de vinculação. Esta validação surge como contributo metodológico relevante, dada a parca investigação debruçada sobre as relações de vinculação no contexto da relação entre irmãos e, em última instância, no contexto das relações familiares.

Os resultados da análise factorial não revelaram a mesma estrutura factorial referida no estudo de Buist e colaboradores (2002) e concordante com as restantes escalas do IPPA (mãe, pai e melhor amigo). No entanto, a estrutura factorial encontrada tem sentido do ponto de vista teórico tendo em conta o estudo da relação entre irmãos. Justificam-se investigações adicionais que corroborem as qualidades psicométricas aqui descritas e permitam a utilização deste instrumento em investigações nacionais futuras que explorem o conceito de vinculação no contexto das relações de fratria.

O segundo estudo focado teve como objectivo examinar as relações entre os estilos de vinculação dos membros de famílias de quatro elementos. Foi possível verificar associações entre os estilos de vinculação dos quatro membros da família, exceptuando apenas no que respeita à relação entre o estilo de vinculação do pai e o estilo de vinculação do filho mais novo. Ainda assim, estes resultados suportam os conceitos de "secure base family" e "family script", ao reflectirem uma congruência entre as percepções dos membros das famílias acerca das suas relações entre si (Byng-Hall, 1995; Byng-Hall \& Stevenson-Hinde, 1991).

A concordância encontrada entre os estilos de vinculação dos membros do casal vai no mesmo sentido de resultados encontrados em outras pesquisas (Batgos \& Leadbeater, 1994; van Ijzendoorn \& Bakermans-Kranenburg, 1996). Quando um casal é constituído por dois membros seguros a hipótese, empiricamente validada, da transmissão intergeracional da vinculação indica-nos que existirá uma maior tendência para que os filhos dessa díade venham a desenvolver um estilo seguro de vinculação, desde que não existam modificações drásticas nos contextos onde estas relações se desenvolvem (Mehta et al., 2009; Soares et al., 2007). O facto de se ter encontrado uma associação significativa entre os estilos de vinculação dos irmãos vai de encontro aos pressupostos teóricos que indicam que as várias relações diádicas da rede familiar se influenciam mutuamente e interagem, criando (ou não) uma base segura comum a todos os membros (Stevenson-Hinde, 1990). A literatura aponta também para a existência de uma concordância entre os estilos de vinculação dos irmãos (Kiang \& Furman, 2007; van Ijzendoorn et al., 2000).

Quanto aos resultados referentes às combinações dos estilos de vinculação das díades, não obstante a predominância das combinações com estilos de vinculação seguros, os dados apontam para o facto de ser mais frequente pais inseguros terem filhos seguros do que pais seguros terem filhos inseguros. O primeiro aspecto poderá reflectir a possibilidade da adolescência, enquanto período de mudanças, poder resultar na descontinuidade da vinculação através 
Geraldes, R., Soares, I. \& Martins, C. (2013). Vinculação no Contexto Familiar: Relações entre Cônjuges, entre Pais e Filhos Adolescentes e entre Irmãos.

do estabelecimento de relações de natureza correctiva; o segundo aspecto, pode ser entendido como reflexo da possibilidade (menos frequente) de existência eventos de vida significativos que justifiquem a descontinuidade da vinculação no sentido da insegurança (Soares et al., 2007).

Como limitação inerente ao estudo, será de realçar que foram analisadas neste estudo as relações de vinculação na família nuclear sob uma perspectiva diádica e individual. Este facto, não permite uma visão integrada de cada agregado familiar. A utilização de instrumentos diversificados, consoante o elemento da família, também poderá ter condicionado a natureza dos resultados obtidos. Neste sentido, torna-se pertinente o investimento, por parte da comunidade científica, em instrumentos de natureza sistémica para a avaliação da qualidade da vinculação no contexto da família nuclear, enquanto unidade social básica (Akister, 1998). Esta possibilidade poderá basear-se nos conceitos, teoricamente relevantes, de "secure base family" e "family script" (Byng-Hall, 1995; Byng-Hall \& Stevenson-Hinde, 1991).

Quanto ao método, a selecção não-aleatória das escolas não permite a generalização dos resultados e o facto do $\mathrm{N}$ amostral de famílias ser reduzido condicionou também as análises de dados efectuadas. Será relevante replicar o presente estudo com uma amostra de famílias mais numerosa, permitindo que outras análises possam ser efectuadas.

Em investigações futuras nesta área, será relevante realizar este estudo com um desenho longitudinal que permita acompanhar as trajectórias desenvolvimentais das relações de vinculação no contexto da família nuclear.

A investigação no domínio da teoria da vinculação e, em particular, a sua aplicação ao contexto da família nuclear é inspiradora. A diversidade de possibilidades que dela decorrem acompanha a diversidade de possibilidades que o ser humano comporta ao longo da sua vida. Assim, as lacunas existentes neste amplo domínio teórico-empírico tornam-se oportunidades únicas de desbravar o ainda vasto campo do desconhecido nesta área. Este estudo abre caminho à investigação acerca da forma como a vinculação se expressa no contexto da família nuclear e, particularmente, no contexto da relação entre irmãos.

\section{Referências}

Ainsworth, M. D. S. (1989). Attachment beyond infancy. American Psychologist, 44(4), 709-716.

Ainsworth, M. D. S., Blehar, M. C., Waters, E., \& Wall, S. (1978). Patterns of attachment. Hillsdale, NJ: Erlbaum.

Akister, J. (1998). Attachment theory and systemic practice: Research update. Journal of Family Therapy, 20, 353-366.

Armsden, G., \& Greenberg, M. (1987). The Inventory of Parent and Peer Attachment: Individual differences and their relationship to psychological well-being in adolescence. Journal of Youth and Adolescence, 16(5), 427-454.

Batgos, J., \& Leadbeater, B. J. (1994). Parental attachment, peer relations and dysphoria in adolescents. In M. B. Sperling \& W H. Berman (Eds.), Attachment in adults: Clinical and development perspectives (pp. 155-178). New York: Guilford Press.
Bowlby, J. (1982). Attachment and loss: Attachment. London: Basic Books (Original work published 1969)

Bretherton, I. (1985). Attachment theory: Retrospect and prospect. In I. Bretherton \& E. Waters (Eds.), Growing points of attachment theory and research. Monographs of the Society for Research in Child Development, 50(1-2, Serial No. 209), 3-35.

Buist, K. L., Dekovic, M., Meeus, W., \& van Aken, M. G. (2002). Developmental patterns in adolescence attachment to mother, father and sibling. Journal of Youth and Adolescence, 31(3), 167-176.

Byng-Hall, J. (1995). Rewriting family scripts: Improvisation and systems change. New York: Guilford Press

Byng-Hall, J., \& Stevenson-Hinde, J. (1991). Attachment relationships within a family system. Infant Mental Health Journal, 12, 187-200.

Canavarro, M. C. (1997). Relações afectivas ao longo do ciclo de vida e saúde mental (Dissertação de doutorado em Psicologia Clínica, Faculdade de Psicologia e Ciências da Educação, Universidade de Coimbra, Portugal).

Canavarro, M. C., Dias, P., \& Lima, V. (2006). A avaliação da vinculação do adulto: Uma revisão crítica a propósito da aplicação da Adult Attachment Interview Scale-R (AAS-R) na população portuguesa. Psicologia (Lisboa), 20(1), 155-185.

Carvalho, M., Soares, I., \& Baptista, A. (2013). The inventory of attachment in childhood and adolescence: Development and psychometric properties. European Journal of Psychological Assessment. Manuscript submitted for publication.

Collins, N., \& Read, S. (1990). Adult attachment relationships, working models and relationships quality in dating couples. Journal of Personality and Social Psychology, 58, 644-683.

Cunha, A. I. (2003). Anorexia nervosa: Vivências e percepções sobre a família (Dissertação de mestrado em Psicologia Clínica, Faculdade de Psicologia e Ciências da Educação, Universidade de Coimbra, Portugal).

Day, R. D., \& Padilla-Walker, L. M. (2009). Mother and father connectedness and involvement during early adolescence. Journal of Family Psychology, 23(6), 900-904.

Fortuna, K., Roisman, G., Haydon, K. C., Groh, A. M., \& Holland, A. S. (2011). Attachment states of mind and the quality of young adults sibling relationships. Developmental Psychology, 47(5), 1366-1373.

Geada, M. (1990). Padrões de vinculação afectiva e níveis de desenvolvimento do auto-conhecimento em toxicodependentes e não-toxicodependentes. Jornal de Psicologia, 9(4-5) 14-18.

Greenberg, M., Siegel, J., \& Leitch, C. (1983). The nature and importance of attachment relationships to parents and peers during adolescence. Journal of Youth and Adolescence, 12, 373-386.

Howe, N., \& Rinaldi, C. M. (2004). 'You be the big sister': Maternal-preschooler internal state discourse, perspective-taking, and sibling caretaking. Infant and Child Development, 13, 217-234.

Kiang, L., \& Furman, W. (2007). Representations of attachment to parents in adolescent siblings pairs: Concordant or discordant? New Directions for Child and Adolescent Development, 117, 73-89.

Marvin, R. S., \& Stewart, R. B. (1990). A family systems framework for the study of attachment. In M. T. Greenberg, D. Cicchetti, \& E. M. Cummings (Eds.), Attachment in the pre-school years: Theory, research and intervention (pp. 51-86). Chicago, IL: University of Chicago Press. 
Mehta, N., Cowan, P. A., \& Cowan, C. P. (2009). Working models of attachment to parents and partners: Implications for emotional behavior between partners. Journal of Family Psychology, 23(6), 895-899.

Neves, L., Soares, I., \& Silva, M. C. (1999). Inventário da vinculação na adolescência - I.P.P.A. In L. Almeida, M. M. Gonçalves, \& M. Simões (Eds.), Testes e provas psicológicas em Portugal: Vol. 2 (pp. 37-48). Braga, Portugal: Associação dos Psicólogos Portugueses.

O'Connor, T. G., \& Croft, C. (2001). A twin study of attachment in preschool children. Child Development, 72(5), 1501-1511.

O'Connor, T. G., Croft, C., \& Steele, H. (2000). The contributions of behavioral genetic studies to attachment theory. Attachment and Human Development, 1, 107-122.

Riggs, S. A., \& Riggs, D. S. (2011). Risk and resilience in military families experiencing deployment: The role of the family attachment network. Journal of Family Psychology, 25(5), 675-687.

Samek, D. R., \& Rueter, M. A. (2011). Considerations of elder sibling closeness in predicting younger sibling substance use: Social learning versus social bonding explanations. Journal of Family Psychology, 25(6), 931-941.

Soares, I., Martins, E. C., \& Tereno, S. (2007). Vinculação na infância. In I. Soares (Ed.), Relações de vinculação ao longo do desenvolvimento: Teoria e avaliação (pp. 47-98). Braga, Portugal: Psiquilíbrios.

Stevenson-Hinde, J. (1990). Attachment within the family systems: An overview. Infant Mental Health Journal, 11(3), 218-229.

Stewart, R. B. (1983). Sibling attachment relationships: Child-infant interactions in the strange situation. Developmental Psychology, 19(2), 192-199.

Sullivan, S. J., Diener, M. L., Mangelsdort, S. C., Brown, G. L, McHale, J. L., \& Frosch, C. A. (2006). Attachment and sensivity in family context: The roles of parent infant gender. Infant Child Development, 15, 367-385.

Teti, D. M., \& Ablard, K. E. (1989). Security of attachment and infant-sibling relationships: A laboratory study. Child Development, 60, 1519-1528.

van Ijzendoorn, M. H. (1992). Intergenerational transmission of parenting: A review of studies in nonclinical populations. Developmental Review, 12, 76-100.

van Ijzendoorn, M. H., \& Bakermans-Kranenburg, M. J. (1996). Attachment representations in mother, father, adolescents and clinical groups: A meta-analytic search for normative data. Journal of Consulting and Clinical Psychology, 64(1), 8-21.

van Ijzendoorn, M. H., Moran, G., Belsky, J., Pederson, D., Bakermans-Kranenburg, M. J., \& Kneppers, K. (2000). The similarity of siblings' attachment to their mother. Child Development, 71(4), 1086-1098.

Volling, B. L. (2001). Early attachment relationships as predictor of preschool children's emotion regulation with a distressed sibling. Early Education and Development, 12(2), 185-207.

Waters, E., \& Cummings, E. M. (2000). A secure base from which to explore close relationships. Child Development, 71(1), 164-172. 